\title{
RISK THEORY AND WIENER PROCESSES
}

\section{H. BoHMAN \\ Stockholm}

We will in this paper consider the risk process from the point of view of random walk in one dimension. The particle starts out at the origin. Each claim is equivalent to a step in the random walk. The length of the step is equal to the amount of the claim minus the amount of the premium which has been obtained since the preceding claim. If the difference is positive the particle advances to the right and if the difference is negative to the left. At distance $U$ to the right from the origin there is a barrier. The problem is to find the distribution function of $X$, the time it takes the particle to cross the barrier for the first time.

In most practical applications of risk theory $U$ is large in comparison to the individual steps of the particle. We will in this paper assume that $U$ is large in comparison to the individual steps and draw certain conclusions about the risk processes from this assumption.

The individual steps of the particle have a certain distribution. The corresponding characteristic function is $\phi$. For reasons which will be seen later we will consider $\phi$ to be a function of it $=\theta$ instead of $t$. This means that

$$
\phi(\theta)=\int_{-\infty}^{+\infty} e^{\theta x} d F(x)
$$

The mean value and the standard deviation of each step is equal to $m$ and $\sigma$ respectively. We now write

$$
\phi(\theta)=\exp \{K(\theta)\}=\exp \left\{m \theta+\frac{\sigma^{2} \theta^{2}}{2}+\ldots\right\}
$$

We now define two random variables $X$ and $Y$.

$$
\begin{aligned}
& X=\text { time to cross the barrier for the first time } \\
& Y=X \sigma^{2} / U^{2} .
\end{aligned}
$$


If $U$ is large in comparison to the individual steps we can apply Wald's approximation to get the characteristic function of the random variable $Y$. This characteristic function is found to be

$$
e^{-\theta(t)}
$$

where $\theta(t)$ is a root of the equation

$$
K(\theta / U)=-i t \sigma^{2} / U^{2}
$$

The reader is referred to [I p. I7-20] or [ 2 p. 568].

We will now make use of our assumption that $U$ is large. More precisely we will assume that

$$
\begin{aligned}
& U / \sigma \text { tends to infinity } \\
& m U / \sigma^{2} \text { remains finite. }
\end{aligned}
$$

The last assumption corresponds to the fact that the security loading $m$ is small in comparison to $U$. We denote

$$
m U / \sigma^{2}=\alpha
$$

We now let $U$ tend to infinity and then get the following equation for $\theta$

$$
\alpha \theta+\theta^{2} / 2=-i t
$$

This equation has two roots. For $t=0$ these two roots become equal to 0 and $-2 \alpha$. For our purpose we have to choose the root which for $t=0$ is equal to the larger of the said two values. This means that

$$
\theta=-\alpha+\sqrt{\alpha^{2}-2 i t}
$$

where the square root is taken with positive real part. We have now found the characteristic function of $Y$, namely

$$
\exp \left\{\alpha-\sqrt{\left.\alpha^{2}-2 i t\right\}}\right.
$$

This is a characteristic function which is well-known from the theory of Wiener processes. The corresponding distribution function is equal to

$$
P(Y<x)=G(\alpha, x)=\int_{0}^{x} \frac{I}{\sqrt{2 \pi}} z^{-3 / 2} e^{-\frac{(1-\alpha z)^{2}}{2 z}} d z
$$


We are now ready to introduce the standard notation $\psi(U, T)$ for ruin probability, equal to the probability that the particle crosses the barrier before time $T$. It follows that we get

$$
\psi(U, T)=G\left(\alpha, T \sigma^{2} / U^{2}\right)
$$

For $\alpha=0$, corresponding to the case that the security loading of the process is zero, the values of $G(o, x)$ are easily obtained from a table of the normal distribution. For $\alpha$ different from zero the values of $G(\alpha, X)$ have to be calculated numerically.

From the expression for the characteristic function we find the values of the probability of ultimate ruin

$$
\begin{array}{ll}
\psi(U, \infty)=\mathrm{I} & \text { if } m \geqslant 0 \\
\psi(U, \infty)=\mathrm{e}^{2 \alpha} & \text { if } m<0
\end{array}
$$

Example I: A company has ro,ooo claims per year. The mean claim amount is equal to $\mathrm{I}, 000$ monetary units. The standard deviation is equal to I0,000 monetary units. The security loading $m$ is equal to zero. The company wants to safeguard with probability 0.99 that ruin will not occur during the first 25 years of operation. We then have

$$
\begin{array}{ll}
\sigma & =10,000 \\
m & =0 \\
T & =250,000 \\
\psi(U, T) & =0.0 \mathrm{I} \\
T \sigma^{2} / U^{2} & =0.15 \mathrm{I}
\end{array}
$$

It follows that $U$ must be equal to 13 million monetary units. As the yearly net premium income is equal to xo million monetary units we find that a risk reserve equal to $130 \%$ of the yearly net premium income is necessary to safeguard survival of the company during the first 25 years of operation with $99 \%$ probability.

Example 2: The same assumptions are made as in example I except that the size of the company is assumed to be ten times larger. This means that the company has I00,000 claims per year and that the yearly net premium income is equal to roo million monetary units. In this case we find that $U$ has to be $4 \mathrm{I}$ million 
monetary units meaning that $U$ has to be $4 \mathrm{I} \%$ of the yearly net premium income.

\section{REFERENCES}

[I] Bartlett, M. S. I955. An introduction to stochastic processes with special reference to methods and applications. The University Press, Cambridge.

[2] Feller, W. I966. An introduction to probability theory and its applications, vol. II. Wiley, New York. 